\title{
Cognitive Improvement Via Left Angular Gyrus-Navigated Repetitive Transcranial Magnetic Stimulation Inducing the Neuroplasticity of Thalamic System in Alzheimer's Disease Spectrum Patients
}

Zhiyuan Yang

Nanjing Drum Tower Hospital: Nanjing University Medical School Affiliated Nanjing Drum Tower Hospital

Xiaoning Sheng

Nanjing Drum Tower Hospital: Nanjing University Medical School Affiliated Nanjing Drum Tower Hospital

Ruomeng Qin

Nanjing Drum Tower Hospital: Nanjing University Medical School Affiliated Nanjing Drum Tower Hospital

Haifeng Chen

Nanjing Drum Tower Hospital: Nanjing University Medical School Affiliated Nanjing Drum Tower Hospital

Pengfei Shao

Nanjing Drum Tower Hospital: Nanjing University Medical School Affiliated Nanjing Drum Tower Hospital

Hengheng Xu

Nanjing Drum Tower Hospital: Nanjing University Medical School Affiliated Nanjing Drum Tower Hospital

Weina Yao

Nanjing University of Traditional Chinese Medicine: Nanjing University of Chinese Medicine

Hui Zhao

Nanjing Drum Tower Hospital: Nanjing University Medical School Affiliated Nanjing Drum Tower Hospital

Yun Xu

Nanjing Drum Tower Hospital: Nanjing University Medical School Affiliated Nanjing Drum Tower Hospital

Feng Bai ( $\sim$ baifeng515@126.com)

Nanjing Drum Tower Hospital: Nanjing University Medical School Affiliated Nanjing Drum Tower Hospital

\section{Research Article}

Keywords: Alzheimer's disease, Neuro-navigated repetitive transcranial magnetic stimulation, Neuroplasticity, Thalamic system

Posted Date: August 24th, 2021

DOI: https://doi.org/10.21203/rs.3.rs-812265/v1

License: @ (i) This work is licensed under a Creative Commons Attribution 4.0 International License. Read Full License 


\section{Abstract}

Background: Stimulating superficial brain regions highly associated with the hippocampus by repetitive transcranial magnetic stimulation (rTMS) may improve memory of Alzheimer disease (AD) spectrum patients.

Methods: We recruited 26 mild cognitive impairment $(\mathrm{MCl})$ and $A D$ patients. All the patients were stimulated to the left angular gyrus, which was confirmed a strong link to the hippocampus through neuroimaging studies, by the neuro-navigated rTMS for four weeks. Automated fiber quantification (AFQ) using diffusion tensor imaging (DTI) metrics and graph theory analysis on functional network were employed to detect the neuroplasticity of brain networks.

Results: After neuro-navigated rTMS intervention, the episodic memory and language function of patients were significantly improved. Increased white matter integrity of right anterior thalamic radiation among $\mathrm{MCl}$ patients, while decreased functional network properties of thalamus subregions were observed. It is worth noting that the improvement of cognition was associated with the neuroplasticity of thalamic system

Conclusions: We speculated that the rTMS intervention targeting left angular gyrus may be served as a strategy to improve cognitive impairment in AD spectrum patients, supporting by the neuroplasticity of thalamic system, especially in the early disease process at the stage of $\mathrm{MCl}$.

\section{Background}

Alzheimer's disease (AD), characterized by the gradually progressive neurodegenerative process, is the most common cause of dementia in the elderly[1]. Currently, treatments for AD mainly focus on drugs aiming at delaying cognitive decline and reversing or affecting the disease's progression. However, the effectiveness of these drugs did not live up to expectations and many clinical trials have failed[2]. It's urged to seek alternative treatment strategies and non-pharmacological interventions.

Repetitive transcranial magnetic stimulation (rTMS) is a non-invasive intervention to the brain, which can excitability and modulate activity across cortico-subcortical networks in the brain[3]. It has been demonstrated that rTMS may be involved in the regulation of neuroplasticity[4] and had opportunities to target specific brain regions and networks[5]. In the development of AD, there are salient neuropathological abnormalities in hippocampus[6, 7]. However, rTMS cannot directly stimulate the deep hippocampus. Recent studies showed that stimulating specific shallow brain regions which are highly associated with the hippocampus may indirectly alter hippocampal activity[8]. According to the calculation of our team (paper unpublished), the region exhibited significant functional connectivity differences among AD spectrum patients is located in the left angular gyrus (MNI: $-45,-67,38$; these data were not listed in this study). Therefore, the left angular gyrus as a potential novel therapeutic target to investigate the efficacy of rTMS in patients with $A D$ and mechanism behind rTMS is worth exploring.

The previous studies have shown strong structural and functional connectivity between the thalamus and the hippocampus[9]. Although thalamus is the most important sensory conduction replacement station[10], and increasing evidence shows that and thalamus plays an important role in episodic memory[11]. Moreover, thalamus is a key neural activity of rTMS affecting episodic memory[12]. Therefore, we speculated that the thalamus and associated network play a vital role in the neural mechanism of enhancing cognition by rTMS in AD.

Diffusion tensor imaging (DTI) is widely used to effectively detect microstructural integrity of white matter [13]. Automated fiber quantification (AFQ) of DTI, is a new method that can recreate whole-brain white matter tracts and estimate point-wise diffusion parameters aimed at the specific tract, which is very sensitive to the white matter abnormalities at the individual level[14]. Previous studies had demonstrated that the volume of fiber tract of thalamus can effectively distinguish between mild cognitive impairment $(\mathrm{MCl})$ and healthy control[15] and white matter integrity in right thalamus was decreased in both $\mathrm{MCl}$ [16] and $\mathrm{AD}[17]$.Meanwhile, restingstate functional magnetic resonance imaging (rs-fMRI) analysis enable us to further understand the mechanism of functional connectivity during rTMS intervention. Functional connectivity in thalamus was reduced [18] and the connection between default mode network and thalamus was significant changed in MCI[19].

To our knowledge, this is the first study to address that neuro-navigated rTMS of the left angular gyrus maybe a novel intervention strategy to improve cognitive impairments in $A D$ spectrum patients. In this study, we examined structural and functional change in $A D$ spectrum patients after four-weeks neuro-navigated rTMS, using AFQ tractography method of white matter tracts and graph theory analysis in functional network. We were especially interested in determining whether the neuroplasticity of thalamic system could be Loading [MathJax]/jax/output/CommonHTML/jax.js g mechanism was further explored. 


\section{Materials And Methods \\ Participants}

The current study was approved by the Ethics Committee of Nanjing Drum Tower Hospital, and written informed consent was obtained from all patients before entering the study. Twenty-six patients, admitted to the Neurology Department in Drum Tower Hospital of Medical School, Nanjing University, were screened for the current study. Two patients were excluded because of excessive head movement during MRI scanning and two patients were excluded because of loss of imaging data. These participants were composed of $\mathrm{MCl}$ and $A D$ patients. The $A D$, in the presence of $A D$ pathology as supported by cerebrospinal fluid or other imaging biomarker, was diagnosed based on the National Institute of Neurological and Communicative Disorders and Stroke and the AD and Related Disorders Association (NINCDSADRDA) and the Diagnostic and Statistical Manual of Mental Disorders IV criteria (DSM-IV) guidelines[20]. The MCI patients included in this study were diagnosed according to the recommendations of Petersen and described as follows[21]: (1) memory complaint confirmed by the subject and/or an informant; (2) objective cognitive performance documented by an auditory verbal learning test-delayed recall (AVLT-DR) scores below or equal to $1.5 \mathrm{SD}$ of education- and age-adjusted norms; (3) clinical dementia rating (CDR) score $=0.5$; (4) the scores for the Mini-Mental State Examination (MMSE) $\geq 24$; and (5) not sufficient to dementia according to NINCDSADRDA and DSM-IV. Exclusion criteria included brain tumors, epilepsy, Parkinson's disease, serve anxiety and depression, thyroid dysfunction or other neurological or psychiatric disorders which can cause memory loss. Participants were excluded if the MRI scans evidenced significant vascular pathology or micro bleeds, or head motion artefacts that affect T1w3d quality and segmentation. Four patients were excluded for these reasons.

\section{Experiment design and Neuro-navigated rTMS}

In the first visit, patients underwent a complete clinical investigation, including medical history and neurological examination, a neuropsychiatric evaluation, brain MRI scanning, and an extensive neuropsychological assessment exploring all cognitive domains. The region, which exhibited significant functional connectivity differences among healthy controls, $\mathrm{MCl}$ and $\mathrm{AD}$ participants, calculated by our team was located at the left angular gyrus (MNI: -45, -67,38). The region was calculated by seed-based functional connectivity analysis using the left hippocampus as a seed. All the patients were stimulated the angular gyrus by the Neuro-navigated rTMS for four weeks. rTMS was applied daily at the same 5 times per week. Neuropsychological measurement and brain MRI scanning were performed again after four weeks rTMS treatment. Details of the study design are summarized in Fig. 1.

rTMS was delivered using a commercially available magnetic stimulator (CCY-IV model; YIRUIDE Inc., Wuhan, China) with a 70-mm figure eight coil and an electromyography device. Each stimulation session consisted of forty circulations of 2 second delivered at $20 \mathrm{~Hz}$ spaced-out by $28 \mathrm{~s}$ of no stimulation, for a grand total of 1600 stimulations. During the rTMS treatment, the coil was set on the angular gyrus was constantly motored using a navigation system, which was anatomically referred by individual T1-weighed MRI volumes. The treatments lasted about 20 minutes. Intensity of stimulation was set at $100 \%$ of the resting motor threshold (RMT), defined as the lowest intensity producing MEPs of $>50 \mu \mathrm{V}$ in at least five out of 10 trials in the relaxed first dorsal interosseous (FDI) muscle of the right hand. RMT was assessed over the optimal stimulus site to elicit MEPs in the right FDI, which was considered motor spot. For each patient, a source estimation on pre-processed TMS data was run at the beginning of each treatment session to confirm the correct anatomical targeting for rTMS.

\section{MRI scanning}

All participants were examined on a Philips 3.0-T scanner (Philips Medical Systems). The examination protocol included the highresolution T1-weighted turbo gradient echo sequence (repetition time $[T R]=9.8 \mathrm{~ms}$, flip angle $[F A]=8^{\circ}$, echo time $[T E]=4.6 \mathrm{~ms}, F O V=250$ $\times 250 \mathrm{~mm}^{2}$, number of slices $=192$, acquisition matrix $=256 \times 256$, thickness $\left.=1.0 \mathrm{~mm}\right)$, the FLAIR sequence $(\mathrm{TR}=4.500 \mathrm{~ms}, \mathrm{TE}=333$ $\mathrm{ms}$, time interval $[\mathrm{TI}]=1.600 \mathrm{~ms}$, number of slices $=200$, voxel size $=0.95 \times 0.95 \times 0.95 \mathrm{~mm} 3$, acquisition matrix $=270 \times 260)$, and the diffusion-weighted imaging sequence (TR $=9.154 \mathrm{~ms}, T E=55 \mathrm{~ms}$, acquisition matrix $=112 \times 112$, FOV $=224 \times 224 \mathrm{~mm} 2$, thickness $=2.5$ $\mathrm{mm}$, voxel size $=2 \times 2 \times 2.5 \mathrm{~mm} 3$, the number of gradient directions $=32(\mathrm{~b}=1000 \mathrm{~s} / \mathrm{mm} 2)$ and one b0 image $)$.

\section{Neuropsychological Measurement}

To evaluate the behavioral effects of the rTMS treatment, we employed a standardized neuropsychological test protocol, including global cognitive assessments and multiple cognitive domain examinations. We also completed the Clinical Dementia Rating Scale (CDRS) to assess the degree of cognitive impairment of the participants. Global cognitive function was evaluated by Mini-Mental State Examination (MMSE) and Montreal Cognitive Assessment Beijing (MoCA-BJ). The raw test scores were converted to Z-scores, which Loading [MathJax]/jax/output/CommonHTML/jax.js 
were used to calculate the compound cognitive index. Episodic memory was calculated as the mean of the Z-scores from Auditory Verbal Learning Test-delayed recall (AVLT-DR) scores and the Wechsler Memory Scale-Visual Reproduction-delayed recall (VR-DR). Information processing speed was calculated as the average Z-scores of the Trail Making Test-A (TMT-A) and the Stroop Color and Word Tests A and B (Stroop A and B). The language function consisted of the Boston Naming Test and Category Verbal Fluency test. Executive function is a compound score of the average Z-scores of the Digit Span Test-backward, Trail Making Test-A (TMT-B) and Stroop Color and Word Tests C (Stroop C). Visuospatial function is a compound score that includes the mean of the Z-scores of the Clock Drawing Test and Visual Reproduction-copy test.

\section{Multimodal magnetic resonance image preprocessing}

In recent years, more and more neuroimaging studies suggested that white matter alterations may be an important pathophysiological feature and a potential target of $A D[22]$. However, whether patterns of white matter change in different fiber tracts are different and what happens to the white matter after the intervention are still largely unknown[13]. We decided to use AFQ, applying deterministic tractography approach, to reconstruct whole-brain white matter and analyze point-wise diffusion parameters in specific fiber tracts. AFQ can not only trace the fiber tracts associated with the thalamus, but also analyze the important tracts in the brain, such as corticospinal tract, cingulate fasciculus, uncinate fasciculus, and arch fasciculus and so on to provide a comprehensive detection for whole-brain[23]. However, the changes in the microstructure of the white matter tracts are not necessarily consistent with alterations in the brain's complex networks[24] and it is better to combine multi-modality data to detect complex network changes in AD patients then a single modality $[25,26]$. It is effective to quantify the complex brain network topology by graph theory using rs-fMRI to build functional network[27, 28]. As a result, we combined DTI and rs-fMRI to better explore thalamus and related network alteration after treatment.

For diffusion images, the data preprocessing was carried out by FSL 5.0.9 software (Oxford Centre for Functional Magnetic Resonance Imaging of the Brain, University of Oxford; https://www.fmrib.ox.ac.uk/fsl/). The preprocessing included the following steps: DICOM-to NIfTI format conversion, registering DWI images $(b=1000 \mathrm{~s} / \mathrm{mm} 2)$ to the non-DWI image (B0), eddy current and head motion correction, and then nonbrain tissue exclusion. After preprocessing, using DTIFIT command of FSL to obtain the whole brain images of diffusion metrics, including fractional anisotropy (FA), mean diffusivity (MD), axial diffusivity (DA) and radial diffusivity (RD), Specific calculation indexes are as follows:

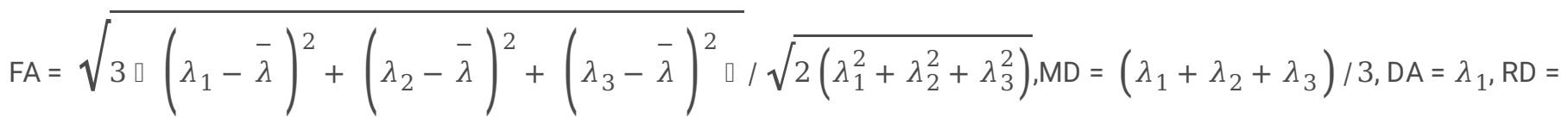

$\left(\lambda_{2}+\lambda_{3}\right) / 2, \lambda_{1}, \lambda_{2}, \lambda_{3}$ reflect the three dispersion directions of water molecules[13]. $\lambda_{1}$ travels in or against the direction of the fiber bundle of the voxel, which called axial direction and $\lambda_{2}, \lambda_{3}$, corresponds to the direction perpendicular to the axis, which called radial direction.

For rs-fMRI images, the data were preprocessed by GRETNA, a graph theoretical network analysis toolbox for imaging connectomes[29]. During the preprocessing, the first 10 volumes for signal were removed to reach a steady state, leaving 220 functional volumes for each participant. The remaining functional volumes were corrected for acquisition time delay between slices (slice timing) and head motion between volumes (realignment). Then, these functional data were normalized to the T1 segmentation individually and spatially smoothed with a Gaussian kernel (full width at half-maximum of $4 \mathrm{~mm}$ ). We regressing out covariates (white matter, cerebral spinal fluid, global signals, and head-motion profiles) by multiple regression analysis to avoid noise signals. Other steps in preprocessing consisting of temporally linear detrending, temporal band-pass filtering $(0.01-0.1 \mathrm{~Hz})$, and scrubbing to reduce the effects of head motion on rs-fMRI data. The network construction was based on a voxel or region of interest approach. The Human Brainnetome Atlas was used to parcellate the brain into 246 regions. All network analyses were performed using GRETNA.

\section{Automated fiber quantification procedure}

We identified 20 major tracts in whole brain and further quantified the diffusion metrics along the tract trajectory by applying the AFQ package. This is a description of AFQ steps in this result:

(1) Fiber Tract Identification

First, 3D T1-weighted images were co-registered into the b0 image for each participant based on FSL, and poorly aligned images were excluded by visual evaluation. Second, using deterministic tractography and a fourth-order Runge-Kutta path integration method[30] to perform whole-brain tractoaraphv with thresholds of turning angle $<30^{\circ}$ and $F A>0.2$. The tracking procedure generates a database of Loading [MathJax]/jax/output/CommonHTML/jax.js

Page $4 / 18$ 
candidate fibers in the whole-brain, which can be broken down into anatomically defined bundles; Third, based on the waypoint ROI procedure described in Wakana et al[31], fiber tract is segmented. In this procedure, if they pass through two waypoints defined by ROI of $A F Q$, fibers are assigned to a specific fiber group. Fourth, by comparing each candidate fiber to fiber tract probability maps, the fiber tract refinement is accomplished. Each fiber conforms to the shape of the tracts defined by the fiber tract probability maps

(2) Fiber Tract Cleaning.

Due to the noise in the data, areas with complex fiber orientation and ambiguous stopping criteria, a few fibers may differ from the rest of the fiber group. The fibers were resampled to 100 equidistant nodes firstly and the fiber tract core is calculated as the mean of each fibers $x, y, z$ coordinates at each node. The spread of fibers in 3-dimensional space is calculated by computing the covariance between each fiber's $x, y, z$ coordinates at 100 nodes. Thus, each node on the tract is represented as a mean coordinate, $m$, and a 3 by 3 covariance matrix, $S$. Then we can calculate its Mahalanobis distance $D_{m}(x)$. The specific formula is as follows: $D_{m}(x)=$ $\sqrt{(x-\mu)^{T} S^{-1}(x-\mu)^{T}}$

$D_{m}(x)$ corresponds to the probability that a given point belongs to the distribution. Abnormal fibers are removed if fibers deviate substantially from the average position.

(3) Fiber Tract Quantification

The fiber group is clipped to the central portion that spans between the two defining ROIs and each fiber was resampled to 100 equally spaced nodes. The properties such as DA, FA, MD and RD at each fiber node are summarized by a weighted average of the diffusion properties. This probability is calculated based on the fiber's Mahalanobis distance from the fiber tract core.

The identified 20 WM tracts in the whole brain are listed in supplementary Table 1. It did not succeed in identifying 20 white matter tracts per participant because of the strict criteria applied by AFQ in the identification of white matter tracts. We excluded 3 fiber tracts, right arcuate fasciculus (AF) and the bilateral cingulum hippocampus (CH) which largely unidentified. Only the remaining 17 fiber tracts would be analyzed in further study. The thresholds were set at a $p<0.05$.

\section{Network parameter analysis}

Graph theoretical analysis was performed on the interregional connectivity matrix by using GRETNA. The weight network properties were calculated under the threshold set by network sparsity with a range of $0.05-0.5$ step size of 0.05 . GRTNA was used to calculate the global network metrics including global efficiency, global clustering coefficient (Cp), characteristic path length (Lp), and nodal network metrics including node degree centrality (DC), nodal global efficiency (Ne) and nodal shortest path (NIp). The calculating formula and descriptions of these topological properties for a network $\mathrm{G}$ with $\mathrm{N}$ nodes and $\mathrm{V}$ edges are as follows[32]:

1. Characteristic Lp at the level of network is an indicator of overall network connectedness and quantifies the parallel information propagation ability, which can be calculated as $L p(G)=\frac{1}{1 / N(N-1) \sum_{i=1}^{N} \sum_{j \neq 1}^{N} 1 / L_{i j}} L_{i j}$ is the characteristic $L p$ between nodes $i$ and j.

2. Eg is defined as the inverse of the harmonic mean of shortest path between each pair of nodes within the network, which effectively measures the information communication capacity of the whole network and is calculated as $\mathrm{Eg}(\mathrm{G})=\frac{1}{N(N-1)} \sum_{i \neq j \in G} \frac{1}{d_{i j}}{ }^{\prime} \mathrm{d}_{\mathrm{ij}}$ is the shortest $L p$ between node $i$ and $j$ in the network.

3. $\mathrm{Cp}$ at the network level represents the degree of local cliquishness or interconnectedness within the network. It can be calculated as $\mathrm{Cp}(\mathrm{G})=$

4. Node degree centrality is number of links connected to a node. It can be represented as $\mathrm{DC}=\sum_{j \in N} d_{i j}$

5. Nodal Lp (NIp) quantifies the mean distance or routing efficiency between one node and all the other nodes in the network, which calculated as: $\operatorname{Lp}(\mathrm{i})=\frac{1}{N-1} \sum_{i \neq j \notin G} d i j, \mathrm{~d}_{\mathrm{ij}}$ is the shortest $\mathrm{Lp}$ between node $\mathrm{i}$ and $\mathrm{j}$ in the network.

6. Nodal efficiency means the efficiency of parallel information transfer of one node in the network. It can be calculated as: $\mathrm{Ne}(\mathrm{i})=$ $\frac{1}{N-1} \sum_{i \neq j \notin G} \frac{1}{d i j}$, dij is the shortest Lp between node $\mathrm{i}$ and $\mathrm{j}$ in the network.

\section{Statistical analyses}

Loading [MathJax]/jax/output/CommonHTML/jax.js 
To examine the point-wise difference of white matter tracts between baseline and post-treatment, we sorted DTI metrics (FA, MD, DA, and $\mathrm{RD}$ ) of 100 nodes along each white matter tract calculated by AFQ in all patients. Then, paired T test was used to detect the differences in the DTI metrics of each fiber tract. Paired T tests were performed in Gretna's nodal metric comparison toolkit and false discovery rate (FDR) was applied to determine the significance for $p$-values $(p<0.05)$. Within each fiber, we only chose more than or equal to three adjacent nodes corrected by FDR to further analyze [26]. Differences of whole-network and nodal properties in functional network between pretherapy and post-treatment was performed in Gretna toolbox using paired samples T test and FDR corrected $p<0.05$.

Baseline and post-treatment cognitive assessment were compared using paired-samples T test in SPSS software (Version 22). We divided the participants into the $\mathrm{AD}$ group and $\mathrm{MCl}$ group. We would conduct data analysis from the perspective of the whole participant, $\mathrm{AD}$ group and $\mathrm{MCl}$ group respectively. In order to investigate possible relationships between alteration of white matter fiber and cognition change, we tested correlations using the Spearmen coefficient (two-tailed) between the altered diffusion metrics and cognitive change (calculated by using post-treatment data minus the baseline data). The thresholds were set at a $p<0.05$.

\section{Results}

\section{Cognitive function improvement by neuro-navigated rTMS}

Participant characteristics and neuropsychological evaluations were shown in Table 1. Compared with the baseline, general cognition (i.e., MOCA-BJ), episodic memory (i.e., AVLT-DR and VR-DR) and language function (i.e., BNT) showed the significant improvement after four-week neuro-navigated rTMS treatment among all participants. However, no difference between the baseline and post-treatment was observed in information processing speed and executive function in these subjects $(p>0.05)$.

Table1

Baseline demographic and neuropsychological data 
Demographics

\begin{tabular}{|c|c|c|c|c|c|c|}
\hline Age (y) & $67.38 \pm 7.67$ & & $67.83 \pm 7.41$ & & & \\
\hline Education (y) & $11.56 \pm 2.45$ & & $10.00 \pm 1.55$ & & & \\
\hline $\begin{array}{l}\text { Gender } \\
\text { (male/female) }\end{array}$ & $6 / 10$ & & & & $3 / 3$ & \\
\hline $\begin{array}{l}\text { General } \\
\text { cognition }\end{array}$ & Baseline & Post-Tx & $p$ & Baseline & Post-Tx & $p$ \\
\hline MMSE & $25.63 \pm 3.18$ & $26.81 \pm 3.12$ & 0.154 & $22.83 \pm 6.89$ & $23.83 \pm 6.28$ & 0.348 \\
\hline MoCA-BJ & $21.88 \pm 3.67$ & $24.13 \pm 2.99$ & $0.002 *$ & $16.17 \pm 7.08$ & $20.67 \pm 7.06$ & $0.007 *$ \\
\hline
\end{tabular}

Composition Z scores of each cognitive domain

\begin{tabular}{|c|c|c|c|c|c|c|c|c|}
\hline $\begin{array}{l}\text { Episodic } \\
\text { Memory }\end{array}$ & & $-0.10 \pm 0.76$ & $0.54 \pm 0.96$ & $<0.001^{\star}$ & $-0.79 \pm 0.66$ & $-0.38 \pm 0.83$ & 0.351 & \\
\hline AVLT-DR & & & $-0.14 \pm 0.53$ & $0.53 \pm 1.10$ & $<0.001^{\star}$ & $-0.75 \pm 0.56$ & $-0.29 \pm 0.81$ & 0.323 \\
\hline VR-DR (WMS) & & & $-0.06 \pm 0.86$ & $0.53 \pm 0.99$ & $0.004^{\star}$ & $-0.83 \pm 0.76$ & $-0.47 \pm 0.92$ & 0.415 \\
\hline $\begin{array}{l}\text { Information } \\
\text { Processing } \\
\text { Speed }\end{array}$ & $0.07 \pm 0.91$ & $0.23 \pm 0.72$ & 0.289 & $-0.42 \pm 0.70$ & $-0.08 \pm 0.56$ & & 0.644 & \\
\hline $\begin{array}{l}\text { TMT-A } \\
\text { (inverse) }\end{array}$ & & & $-0.04 \pm 0.90$ & $0.22 \pm 0.97$ & 0.100 & $-0.23 \pm 1.06$ & $-0.25 \pm 1.38$ & 0.956 \\
\hline $\begin{array}{l}\text { Stroop A } \\
\text { (inverse) }\end{array}$ & & & $0.08 \pm 1.17$ & $0.28 \pm 0.97$ & 0.242 & $-0.57 \pm 0.56$ & $-0.37 \pm 0.71$ & 0.225 \\
\hline $\begin{array}{l}\text { Stroop B } \\
\text { (inverse) }\end{array}$ & & & $0.16 \pm 1.35$ & $0.18 \pm 0.77$ & 0.912 & $-0.47 \pm 0.56$ & $-0.46 \pm 0.57$ & 0.904 \\
\hline Language & & & $-0.06 \pm 0.68$ & $0.20 \pm 0.71$ & $0.017 *$ & $-0.37 \pm 0.75$ & $0.00 \pm 0.42$ & 0.065 \\
\hline CVF & & & $0.13 \pm 0.86$ & $0.36 \pm 1.03$ & 0.230 & $-0.76 \pm 1.10$ & $-0.55 \pm 0.70$ & 0.415 \\
\hline BNT & & & $-0.27 \pm 1.14$ & $0.05 \pm 1.05$ & $0.004^{*}$ & $0.01 \pm 0.77$ & $0.54 \pm 0.46$ & $0.024^{*}$ \\
\hline $\begin{array}{l}\text { Executive } \\
\text { Function }\end{array}$ & & & $0.03 \pm 0.74$ & $0.18 \pm 0.75$ & 0.185 & $-0.14 \pm 0.80$ & $-0.08 \pm 0.56$ & 0.648 \\
\hline DST-backward & & & $-0.03 \pm 0.77$ & $0.34 \pm 1.20$ & $0.034^{\star}$ & $-0.73 \pm 1.12$ & $-0.08 \pm 0.49$ & 0.144 \\
\hline $\begin{array}{l}\text { TMT-B } \\
\text { (inverse) }\end{array}$ & & & $-0.10 \pm 0.81$ & $0.06 \pm 0.83$ & 0.136 & $0.14 \pm 1.66$ & $0.01 \pm 1.55$ & 0.751 \\
\hline $\begin{array}{l}\text { Stroop C } \\
\text { (inverse) }\end{array}$ & & & $0.23 \pm 1.25$ & $0.14 \pm 0.85$ & 0.672 & $-0.47 \pm 0.73$ & $-0.51 \pm 0.62$ & 0.768 \\
\hline
\end{tabular}

Note: Values are presented as the mean \pm standard error (SE).

Abbreviations: AVLT-DR, Auditory Verbal Learning Test-delayed recall; BNT, Boston Naming Test; CVF, category verbal fluency; DST, Digit Span Test;

MMSE, mini mental state examination; MoCA-BJ, Beijing version of the Montreal Cognitive Assessment; Stroop A, B and C, Stroop Color and Word Tests A, B, and C; TMT-A and TMT-B, Trail Making Test-A and B; VR-DR, visual reproduction-delay recall;

*Indicates a statistical difference between groups, $p<0.05$.

After dividing all participants into $\mathrm{AD}$ and $\mathrm{MCl}$, we respectively analyzed cognitive function in these two groups (Table 1 and Figure 2) Wh faund that thn ananitivn imnromamnnt was occurred in general cognition (i.e., MOCA-BJ), episodic memory (i.e., AVLT-DR and VR-DR) Loading [MathJax]/jax/output/CommonHTML/jax.js 
and language function (i.e., BNT) among MCl group $(p<0.05)$. In addition, although there are some differences in neuropsychological tests, there also exist some very similar improvement on general cognition (i.e., MOCA-BJ) and language function (i.e., BNT) in AD group $(p<0.05)$.

\section{Point-wise differences of white matter tracts between baseline and post- treatment}

Group point-wise differences of white matter tract were determined by mean diffusion metrics (FA, MD, $A D$, and RD) and only more than or equal to three adjacent nodes were reported within each fiber. The major tracts we studied were bilateral thalamic radiation, which regions of interests are based on the coronal plane at the anterior edge of pons to delineate thalamus and internal capsule[31]. We also analyzed other fiber tracts successfully tracked as described above. The following were the details (Figure 3).

(i) Total participants: In point-wise comparison of white matter fiber tracts values, we found the intermediate component of the left Cingulum Cingulate's MD (nodes 28-33) and RD (nodes 29-32) values were significantly changed (FDR correction, $p<0.05$ ). After rTMS treatment, these values show an increasing trend.

(ii) $\mathrm{MCl}$ group and $\mathrm{AD}$ group: Among the $\mathrm{MCl}$ group, we found the posterior portion of the right anterior thalamic radiation's $\mathrm{FA}$ (nodes 88 94) values and the intermediate component of the left uncinate fasciculus's FA (nodes 51-54) values had been improved after rTMS treatment. By contrast, the intermediate component of the left Cingulum Cingulate's MD (nodes 29-31) values showed the continuing damage after rTMS treatment. In addition, there was no significant change of point-wise differences of white matter tracts between baseline and post-treatment in AD group. We calculated fiber tract level differences of white matter tracts in AD group by calculating average value of each tract as a complement, and the Callosum Forceps was observed slight changes (supplementary Figure1).

\section{Function network topology properties change in thalamus after treatment}

The region of functional network was based on the Human Brainnetome Atlas and the brain aera representing the thalamus were selected [33]. Thalamus was divided into medial pre-frontal thalamus, pre-motor thalamus, sensory thalamus, rostral temporal thalamus, posterior parietal thalamus, occipital thalamus, caudal temporal thalamus and lateral pre-frontal thalamus[33]. Our study shows significant alterations in the degree centrality (DC), nodal efficiency ( $\mathrm{Ne}$ ) and nodal shortest path (NIp) in patients with $\mathrm{MCl}$. In $\mathrm{MCl}$ group, the $\mathrm{DC}$ and $\mathrm{Ne}$ of right medial pre-frontal thalamus, right posterior parietal thalamus, right occipital thalamus and right lateral pre-frontal thalamus were decreased after treatment. The Ne of caudal temporal thalamus was also decreased in $\mathrm{MCl}$. In the meanwhile, the NIp of right posterior parietal thalamus, right occipital thalamus and right lateral pre-frontal thalamus was increased after treatment (Figure4, $p<0.05$, FDR corrected). We didn't find similar changes in AD patients. In the meanwhile, we analyzed brain regions associated with remaining altered two tracts- left Cingulum Cingulate and left uncinate fasciculus. The brain regions of the analysis were defined according to the starting and ending points of the fiber tracking[31]. The network properties of these brain regions did not change significantly after intervention.

\section{The changed diffusion metrics, theoretical parameter and behavioral significance}

Based on the multi-modal analysis in Figure 3 and Figure 4, we further analyzed the correlation between the diffusion metrics, theoretical parameter and cognition respectively (Figure5):

(i) Post-wise differences correlation: It should be noted that the improved white matter integrity of right Thalamic Radiation were observed after four-week neuro-navigated rTMS treatment. Among $\mathrm{MCl}$ group, the increased white matter integrity of posterior portion of the right Thalamic Radiation's (nodes 88-94) correlated positively with the improved episodic memory $(r=0.585, p=0.028)$ and improved language function $(r=0.663, p=0.007)$

(ii) Theoretical parameter correlation: previous studies demonstrated that compared to healthy control, AD patients showed higher degree centrality and nodal efficiency in thalamus[34]. We thought that network attributes of the thalamus were improved after the intervention. In order to better describe this improvement, we took the negative number of these attributes for correlation analysis. After Loading [MathJax]/jax/output/CommonHTML/jax.js ve correlation between transformed Ne of right posterior parietal thalamus $(r=0.543, p=$ 
$0.030)$, right lateral pre-frontal thalamus $(r=0.497, p=0.050)$ and the improved episodic memory among $\mathrm{MCl}$ group. In addition, we also found similar positive correlation between $\mathrm{DC}(\mathrm{r}=0.577, p=0.021), \mathrm{Ne}(\mathrm{r}=0.674, p=0.004)$ of right posterior parietal thalamus, $\mathrm{Ne}(r=$ $0.726, p=0.001$ ) of right occipital thalamus and the language function (the score of BNT test) among $\mathrm{MCl}$ group. The improved Nlp of right posterior parietal thalamus $(r=0.806, p<0.001)$, right occipital thalamus $(r=-0.824, p<0.001)$ was positively correlated with the score of BNT test.

\section{Discussion}

Our study was the first to use neuro-navigated rTMS targeting left angular gyrus to improve cognitive impairments in AD spectrum patients. And, we firstly demonstrated the changes in fiber associated with thalamus after neuro-navigated rTMS treatment in AD spectrum patients. We investigated function network alteration in patients from a multimodal perspective and found a significant beneficial effect of rTMS targeting angular gyrus on improving episodic memory and language function. Furthermore, significant improvement was observed in microstructural integrity of right anterior thalamic radiation. Network topology properties in thalamus, such as nodal efficiency and degree centrality, were also changed at the function network level. This investigative approach may lead to a better understanding of cognitive improvement via neuro-navigated rTMS inducing the neuroplasticity of thalamic system in AD spectrum patients.

\section{The selection and importance of stimulation target}

In previous studies, rTMS intervention tends to stimulate the brain area traditionally associated with brain function such as dorsolateral prefrontal cortex (DLPFC)[35], inferior frontal gyrus(IFG)[36], parietal lobe and temporal lobe[37] and so on. These conventional interventions are often localized through body surface markers and brain region system such as electroephalogram 10-20 system. These positioning methods are not accurate enough, easy to occur error and other side-effects[38].Although they have a partial intervention effect, it's too crude to further explore the mechanism behind the study. As technology develops, neuro-navigated rTMS has significant advantages, such as more accurate location determination by using structural and functional neuroimaging and choosing target according to specific study purpose[39]. In this study, our team calculated the region which is the most extraordinary different among healthy controls, $\mathrm{MCl}$ and $\mathrm{AD}$ patients using functional connectivity analysis based on the left hippocampus and located it to the left angular gyrus in our other large sample (these data were not listed in this study). Our intervention target was identified precisely and individually by neuro-navigated system. Previous researchers have used this precise navigation technique to target region of interest, such as, Koch et al. treated AD patients with rTMS in the precuneus region (MNI : 0,-65,37) and found their AVLT-DR scores improved significantly[39]; Ilona Eliasova performed intervention in the right inferior frontal gyrus (MNI : 48, 21,3) in AD and MCl patients and resulted that TMT-A and TMT-B scores was improved after treatment[40].

The improvement of memory function by rTMS is consistent with previous studies, that angular gyrus, and its connectivity with the hippocampus, are involved in different degrees of memory function[41]. Recent models of long-term memory showed that the left angular gyrus played a critical role in episodic retrieval and recollection[42-44]. Inhibitory TMS of the angular gyrus impaired retrieval of episodic memory[45, 46], and supported encoding in experience memory [47]. Moreover, memory encoding is an important target of rTMS to improve episodic memory[48]. These results lead us to propose a hypothesis that navigated rTMS targeting angular gyrus improve episodic memory by influencing memory encoding and left angular gyrus-navigated rTMS may be an effective treatment to improve memory in $A D$ patients.

\section{The underlying mechanism and intervention opportunity of cognitive improvement}

Thalamus is traditionally considered as a transmission center, responsible for transmission of sensory and motor inputs to the cerebral cortex, however, recent studies had shown that thalamus also play an important role in memory function[49, 50]. It is consistent with our results that after four-week rTMS intervention, patient's memory improved along with changes in the thalamus. Previous study showed that the white matter integrity of anterior thalamic radiation was damaged in AD spectrum patients[51-53]. These studies indicated that the anterior thalamic radiation may serve as an important marker of AD diagnose. Our results showed that the FA value of right anterior thalamic radiation increased in $\mathrm{MCl}$ group after rTMS treatment. This demonstrated that these white matter fiber bundles may have been remodeled due to the rTMS intervention, and the improvement of episodic memory and language scale score was positively

Loading [MathJax]/jax/output/CommonHTML/jax.js

Page $9 / 18$ 
correlated with the degree of such reconstruction. These findings provide a new underlying mechanism of how rTMS improves cognition.

In addition, to explain the therapeutic mechanism of rTMS from the perspective of white matter integrity, functional network change can't be ignored. It's worth noting that the potential effect of rTMS spread from directly targeted areas to anatomically areas, which provides an opportunity to apply rTMS at one point of neural circuit[54]. And, previous graph theoretical analysis in functional network showed that AD patients had higher degree centrality and nodal efficiency in thalamus[34]. These findings were consistent to our results. By shifting the thalamus FC graph pattern to that of healthy control, rTMS improved MCl's episodic memory and language function. Our correlation analysis also confirmed that the greater the alteration, the better the patient's memory and language function. Interestingly, we also found changes in other fiber tracts, with either an increase or a decrease in the integrity of white matter tracts, which may be the result of natural physiological processes and need furthered investigated. We found these white matter and functional changes primarily in the $\mathrm{MCl}$ population suggested that rTMS has a good effect in the early stages of cognitive impairment. Our findings were consistent with previous studies about the curative effect of rTMS in which patients with mild AD showed better cognitive improvement than those with moderate $A D$ through combined rTMS and cognitive training intervention[55, 56]. In late AD, irreversible damage has occurred, including excessive $A \beta$ accumulation, neuronal damage, death, and destruction of the blood-brain barrier[57]. At this stage, drugs or other interventions are difficult to play a good therapeutic effect.

Our study showed that after four-week rTMS intervention targeting angular gyrus, memory and language function in both $\mathrm{MCl}$ and $\mathrm{AD}$ patients had been improved. White matter integrity of right anterior thalamic radiation is reconstructed and functional network topology properties was refined in thalamus. These alterations play a key role in cognitive improvement. The causal relationship between these changes will be the focus of future research.

\section{Methodological issues Limitations and prospects}

There are several limitations of our study needs improved. Firstly, these observations in our study were made in a relatively small sample and lacked a sham group due to the relatively complex experimental design. Further investigation through large prospective studies is necessary. Secondly, depending on the strict requirements for fiber tracking, the fiber tracts like bilateral cingulum hippocampus tracts were not traceable in some subjects, led to lack into these important fiber tracts, however, all of our subjects' target fibers associated with thalamus were reconstructed successfully, which lends confidence in further study.

\section{Conclusion}

In support of the growing evidence for an intervention strategy of AD spectrum patients, this study provided neuroimaging evidence that the cognitive improvement via left angular gyrus-navigated rTMS inducing the neuroplasticity of thalamic system, especially in the early disease process at the stage of $\mathrm{MCl}$. These findings may open novel avenues for better understanding the effects of rTMS in improving episodic memory and language function in AD spectrum patients.

\section{Abbreviations}

AD, Alzheimer's disease; AFQ, Automated fiber quantification; AVLT-DR, Auditory Verbal Learning Test-delayed recall; BNT, Boston Naming Test; Cp, global clustering coefficient; CVF, category verbal fluency; DA, axial diffusivity; DC, node degree centrality; DST, Digit Span Test; DTI, Diffusion tensor imaging; FDI, first dorsal interosseous; FDR, false discovery rate; FA, fractional anisotropy; Lp, characteristic path length; MD, mean diffusivity; $\mathrm{MCl}$, mild cognitive impairment; MMSE, mini mental state examination; MoCA-BJ, Beijing version of the Montreal Cognitive Assessment; Ne, nodal global efficiency; NIp, nodal shortest path; RMT, resting motor threshold; RD, radial diffusivity; rs-fMRI, Resting-state functional magnetic resonance imaging; rTMS, Repetitive transcranial magnetic stimulation; Stroop A, B and C, Stroop Color and Word Tests A, B, and C; TMT-A and TMT-B, Trail Making Test-A and B; VR-DR, visual reproduction-delay recall

\section{Declarations}

\section{Ethics approval and consent to participate}

Individuals provided informed consent prior to participation at each measurement occasion. The Ethics Committee of Nanjing Drum

Loading [MathJax]/jax/output/CommonHTML/jax.js

Page 10/18 


\section{Consent for publication}

Not applicable

\section{Availability of data and materials}

The datasets used during the current study are available from the corresponding author on reasonable request.

\section{Competing interests}

The authors declare that they have no conflict of interest.

\section{Funding}

This work was supported partly by grants from the National Natural Science Foundation of China (No. 81822013; 82071186), Jiangsu Provincial Key Medical Talents (No. ZDRCA2016085), the Key Research and Development Program of Jiangsu Province of China (BE2016610), the National Key Research and Development Program of China (2016YFC1300500-504) and Jiangsu Province Key Medical Discipline (ZDXKA2016020).

\section{Authors' contributions}

ZY, XS, RQ, coordinated the study. YX, HZ, FB designed the study. ZY, XS, FC performed the experiments. PS, HX did the MRI scanning. $\mathrm{RQ}, \mathrm{WY}$ employed neuropsychological assessment, $\mathrm{YX}, \mathrm{FB}$ supervised data analyses. $Z Y$ analysed the data and was major contributor in writing the manuscript; All authors approved the final version of the manuscript; FB obtained funding designed to the study

\section{Acknowledgements}

Not applicable

\section{References}

1. Nichols E, Szoeke CEl, Vollset SE, Abbasi N, Abd-Allah F, Abdela J, et al. Global, regional, and national burden of Alzheimer's disease and other dementias, 1990-2016: a systematic analysis for the Global Burden of Disease Study 2016. Lancet Neurol. 2019;18(1):88-106. http://doi.org/10.1016/s1474-4422(18)30403-4.

2. Wisniewski T, Konietzko U. Amyloid- $\beta$ immunisation for Alzheimer's disease. Lancet Neurol. 2008;7(9):805-11. http://doi.org/10.1016/s1474-4422(08)70170-4.

3. Fox MD, Halko MA, Eldaief MC, Pascual-Leone A. Measuring and manipulating brain connectivity with resting state functional connectivity magnetic resonance imaging (fcMRI) and transcranial magnetic stimulation (TMS). Neuroimage. 2012;62(4):2232-43. http://doi.org/10.1016/j.neuroimage.2012.03.035.

4. Weiduschat N, Thiel A, Rubi-Fessen I, Hartmann A, Kessler J, Merl P, et al. Effects of repetitive transcranial magnetic stimulation in aphasic stroke: a randomized controlled pilot study. Stroke. 2011;42(2):409-15. http://doi.org/10.1161/STROKEAHA.110.597864.

5. Shafi M, Westover M, Fox M, Pascual-Leone A. Exploration and modulation of brain network interactions with noninvasive brain stimulation in combination with neuroimaging. Eur J Neurosci. 2012;35(6):805-25. http://doi.org/10.1111/j.14609568.2012.08035.x.

6. Guebel D, Torres N, Acebes Á. Mapping the transcriptomic changes of endothelial compartment in human hippocampus across aging and mild cognitive impairment. Biology open 2021, 10(5). http://doi.org/10.1242/bio.057950.

7. King-Robson J, Wilson H, Politis M. Associations Between Amyloid and Tau Pathology, and Connectome Alterations, in Alzheimer's Disease and Mild Cognitive Impairment. Journal of Alzheimer's disease: JAD 2021. http://doi.org/10.3233/jad-201457. 
8. Guidali G, Pisoni A, Bolognini N, Papagno C. Keeping order in the brain: The supramarginal gyrus and serial order in short-term memory. Cortex. 2019;119:89-99. http://doi.org/10.1016/j.cortex.2019.04.009.

9. Yang C, Ren J, Li W, Lu M, Wu S, Chu T. Individual-level morphological hippocampal networks in patients with Alzheimer's disease. Brain Cogn. 2021;151:105748. http://10.1016/j.bandc.2021.105748.

10. Cunningham S, Tomasi D, Volkow N. Structural and functional connectivity of the precuneus and thalamus to the default mode network. Hum Brain Mapp. 2017;38(2):938-56. http://10.1002/hbm.23429.

11. Savage L, Hall J, Vetreno R. Anterior thalamic lesions alter both hippocampal-dependent behavior and hippocampal acetylcholine release in the rat. Learning \& memory (Cold Spring Harbor, NY) 2011, 18(12):751-758. http://doi.org/10.1101/lm.023887.111.

12. Hebscher M, Kragel JE, Kahnt T, Voss JL. Enhanced reinstatement of naturalistic event memories due to hippocampal-networktargeted stimulation. Curr Biol. 2021;31(7):1428-37 e1425. http://doi.org/10.1016/j.cub.2021.01.027.

13. Meeter LH, Kaat LD, Rohrer JD, van Swieten JC. Imaging and fluid biomarkers in frontotemporal dementia. Nat Rev Neurol. 2017;13(7):406-19. http://doi.org/10.1038/nrneurol.2017.75.

14. Yeatman J, Dougherty R, Myall N, Wandell B, Feldman H. Tract profiles of white matter properties: automating fiber-tract quantification. PloS one. 2012;7(11):e49790. http://doi.org/10.1371/journal.pone.0049790.

15. Lee W, Park B, Han K. Classification of diffusion tensor images for the early detection of Alzheimer's disease. Comput Biol Med. 2013;43(10):1313-20. http://doi.org/10.1016/j.compbiomed.2013.07.004.

16. Rose S, McMahon K, Janke A, O'Dowd B, de Zubicaray G, Strudwick M, et al. Diffusion indices on magnetic resonance imaging and neuropsychological performance in amnestic mild cognitive impairment. J Neurol Neurosurg Psychiatry. 2006;77(10):1122-8. http://doi.org/10.1136/jnnp.2005.074336.

17. Latini F, Fahlström M, Marklund N, Feresiadou A. White matter abnormalities in a patient with visual snow syndrome: New evidence from a diffusion tensor imaging study. European journal of neurology. 2021. http://doi.org/10.1111/ene.14903.

18. Tang F, Zhu D, Ma W, Yao Q, Li Q, Shi J. Differences Changes in Cerebellar Functional Connectivity Between Mild Cognitive Impairment and Alzheimer's Disease: A Seed-Based Approach. Front Neurol. 2021;12:645171. http://doi.org/10.3389/fneur.2021.645171.

19. Gilligan T, Sibilia F, Farrell D, Lyons D, Kennelly S, Bokde A. No relationship between fornix and cingulum degradation and withinnetwork decreases in functional connectivity in prodromal Alzheimer's disease. PloS one. 2019;14(10):e0222977. http://doi.org/10.1371/journal.pone.0222977.

20. McKhann GM, Knopman DS, Chertkow H, Hyman BT, Jack CR Jr, Kawas CH, et al. The diagnosis of dementia due to Alzheimer's disease: recommendations from the National Institute on Aging-Alzheimer's Association workgroups on diagnostic guidelines for Alzheimer's disease. Alzheimers Dement. 2011;7(3):263-9. http://doi.org/10.1016/j.jalz.2011.03.005.

21. Palmqvist S, Janelidze S, Quiroz YT, Zetterberg H, Lopera F, Stomrud E, et al. Discriminative Accuracy of Plasma Phospho-tau217 for Alzheimer Disease vs Other Neurodegenerative Disorders. JAMA. 2020;324(8):772-81. http://doi.org/10.1001/jama.2020.12134.

22. Nasrabady SE, Rizvi B, Goldman JE, Brickman AM. White matter changes in Alzheimer's disease: a focus on myelin and oligodendrocytes. Acta Neuropathol Commun. 2018;6(1):22. http://doi.org/10.1186/s40478-018-0515-3.

23. Yeatman JD, Dougherty RF, Myall NJ, Wandell BA, Feldman HM. Tract profiles of white matter properties: automating fiber-tract quantification. PLoS One. 2012;7(11):e49790. 10.1371/journal.pone.0049790.

24. Momi D, Ozdemir RA, Tadayon E, Boucher P, Shafi MM, Pascual-Leone A, et al. Network-level macroscale structural connectivity predicts propagation of transcranial magnetic stimulation. Neuroimage. 2021;229:117698. http://doi.org/10.1016/j.neuroimage.2020.117698.

25. Lin W, Lin W, Chen G, Zhang H, Gao Q, Huang Y, et al. Bidirectional Mapping of Brain MRI and PET With 3D Reversible GAN for the Diagnosis of Alzheimer's Disease. Front Neurosci. 2021;15:646013. http://doi.org/10.3389/fnins.2021.646013.

26. Pistono A, Senoussi M, Guerrier L, Rafiq M, Gimeno M, Péran P, et al. Language Network Connectivity Increases in Early Alzheimer's Disease. Journal of Alzheimer's disease: JAD. 2021. http://doi.org/10.3233/jad-201584.

27. Li W, Zhao H, Qing Z, Nedelska Z, Wu S, Lu J, et al. Disrupted Network Topology Contributed to Spatial Navigation Impairment in Patients With Mild Cognitive Impairment. Front Aging Neurosci. 2021;13:630677. http://doi.org/10.3389/fnagi.2021.630677.

28. Rauchmann BS, Ersoezlue E, Stoecklein S, Keeser D, Brosseron F, Buerger K, et al. Resting-State Network Alterations Differ between Alzheimer's Disease Atrophy Subtypes. Cereb Cortex. 2021. http://doi.org/10.1093/cercor/bhab130.

Loading [MathJax]/jax/output/CommonHTML/jax.js

Page $12 / 18$ 
29. Wang J, Wang X, Xia M, Liao X, Evans A, He Y. GRETNA: a graph theoretical network analysis toolbox for imaging connectomics. Front Hum Neurosci 2015, 9:386. http://doi.org/10.3389/fnhum.2015.00386.

30. Basser P, Pajevic S, Pierpaoli C, Duda J, Aldroubi A: In vivo fiber tractography using DT-MRI data. Magnetic resonance in medicine 2000, 44(4):625-632. http://doi.org/10.1002/1522-2594(200010)44:4<625::aid-mrm17>3.0.co;2-o

31. Wakana S, Caprihan A, Panzenboeck MM, Fallon JH, Perry M, Gollub RL, et al. Reproducibility of quantitative tractography methods applied to cerebral white matter. Neuroimage. 2007;36(3):630-44. http://doi.org/10.1016/j.neuroimage.2007.02.049.

32. Rubinov M, Sporns O. Complex network measures of brain connectivity: uses and interpretations. Neuroimage. 2010;52(3):105969. http://doi.org/10.1016/j.neuroimage.2009.10.003.

33. Fan L, Li H, Zhuo J, Zhang Y, Wang J, Chen L, et al. The Human Brainnetome Atlas: A New Brain Atlas Based on Connectional Architecture. Cereb Cortex. 2016;26(8):3508-26. http://doi.org/10.1093/cercor/bhw157.

34. Ng ASL, Wang J, Ng KK, Chong JSX, Qian X, Lim JKW, et al. Distinct network topology in Alzheimer's disease and behavioral variant frontotemporal dementia. Alzheimers Res Ther. 2021;13(1):13. http://doi.org/10.1186/s13195-020-00752-w.

35. Ahmed MA, Darwish ES, Khedr EM, El Serogy YM, Ali AM. Effects of low versus high frequencies of repetitive transcranial magnetic stimulation on cognitive function and cortical excitability in Alzheimer's dementia. J Neurol. 2012;259(1):83-92. http://doi.org/10.1007/s00415-011-6128-4.

36. Rubi-Fessen I, Hartmann A, Huber W, Fimm B, Rommel T, Thiel A, et al: Add-on Effects of Repetitive Transcranial Magnetic Stimulation on Subacute Aphasia Therapy: Enhanced Improvement of Functional Communication and Basic Linguistic Skills. A Randomized Controlled Study. Archives of physical medicine and rehabilitation 2015, 96(11):1935-1944.e1932. http://doi.org/10.1016/j.apmr.2015.06.017.

37. Zhao J, Li Z, Cong Y, Zhang J, Tan M, Zhang H, et al: Repetitive transcranial magnetic stimulation improves cognitive function of Alzheimer's disease patients. Oncotarget 2017, 8(20):33864-33871. http://doi.org/10.18632/oncotarget.13060.

38. Buss SS, Fried PJ, Pascual-Leone A. Therapeutic noninvasive brain stimulation in Alzheimer's disease and related dementias. Curr Opin Neurol. 2019;32(2):292-304. http://doi.org/10.2174/1567205013666160930113907.

39. Koch G, Bonni S, Pellicciari MC, Casula EP, Mancini M, Esposito R, et al. Transcranial magnetic stimulation of the precuneus enhances memory and neural activity in prodromal Alzheimer's disease. Neuroimage. 2018;169:302-11. http://doi.org/10.1016/j.neuroimage.2017.12.048.

40. Eliasova I, Anderkova L, Marecek R, Rektorova I. Non-invasive brain stimulation of the right inferior frontal gyrus may improve attention in early Alzheimer's disease: a pilot study. J Neurol Sci. 2014;346(1-2):318-22. http://doi.org/10.1016/j.jns.2014.08.036.

41. Sestieri C, Shulman G, Corbetta M. The contribution of the human posterior parietal cortex to episodic memory. Nature reviews Neuroscience. 2017;18(3):183-92. http://doi.org/10.1038/nrn.2017.6.

42. Berryhill M. Insights from neuropsychology: pinpointing the role of the posterior parietal cortex in episodic and working memory. Front Integr Nuerosci. 2012;6:31. http://doi.org/10.3389/fnint.2012.00031.

43. Ramanan S, Piguet O, Irish M. Rethinking the Role of the Angular Gyrus in Remembering the Past and Imagining the Future: The Contextual Integration Model. The Neuroscientist: a review journal bringing neurobiology, neurology and psychiatry 2018, 24(4):342-352. http://doi.org/10.1177/1073858417735514.

44. Rugg M, King D. Ventral lateral parietal cortex and episodic memory retrieval. Cortex; a journal devoted to the study of the nervous system and behavior 2018, 107:238-250. http://doi.org/10.1016/j.cortex.2017.07.012.

45. Sestieri C, Capotosto P, Tosoni A, Luca Romani G, Corbetta M. Interference with episodic memory retrieval following transcranial stimulation of the inferior but not the superior parietal lobule. Neuropsychologia. 2013;51(5):900-6. http://doi.org/10.1016/j.neuropsychologia.2013.01.023.

46. Thakral PP, Madore KP, Schacter DL. A Role for the Left Angular Gyrus in Episodic Simulation and Memory. The Journal of Neuroscience. 2017;37(34):8142-9. http://doi.org/10.1523/jneurosci.1319-17.2017.

47. Koen JD, Thakral PP, Rugg MD. Transcranial magnetic stimulation of the left angular gyrus during encoding does not impair associative memory performance. Cogn Neurosci. 2018;9(3-4):127-38. http://doi.org/10.1080/17588928.2018.1484723.

48. Hebscher M, Kragel JE, Kahnt T, Voss JL. Enhanced reinstatement of naturalistic event memories due to hippocampal-networktargeted stimulation. Curr Biol 2021:1-10. http://doi.org/10.1016/j.cub.2021.01.027.

49. Schmitt LI, Wimmer RD, Nakajima M, Happ M, Mofakham S, Halassa MM. Thalamic amplification of cortical connectivity sustains attentional control. Nature. 2017:545(7653):219-23. http://doi.org/10.1038/nature22073.

Loading [MathJax]/jax/output/CommonHTML/jax.js

Page 13/18 
50. Bolkan SS, Stujenske JM, Parnaudeau S, Spellman TJ, Rauffenbart C, Abbas Al, et al. Thalamic projections sustain prefrontal activity during working memory maintenance. Nat Neurosci. 2017;20(7):987-96. http://doi.org/10.1038/nn.4568.

51. Chen H, Sheng X, Qin R, Luo C, Li M, Liu R, et al. Aberrant White Matter Microstructure as a Potential Diagnostic Marker in Alzheimer's Disease by Automated Fiber Quantification. Front Neurosci. 2020;14:570123. http://doi.org/10.3389/fnins.2020.570123.

52. Shao W, Li X, Zhang J, Yang C, Tao W, Zhang S, et al. White matter integrity disruption in the pre-dementia stages of Alzheimer's disease: from subjective memory impairment to amnestic mild cognitive impairment. Eur J Neurol. 2019;26(5):800-7. http://doi.org/10.1111/ene.13892.

53. Setiadi TM, Martens S, Opmeer EM, Marsman JC, Tumati S, Reesink FE, et al. Widespread white matter aberration is associated with the severity of apathy in amnestic Mild Cognitive Impairment: Tract-based spatial statistics analysis. Neuroimage Clin. 2021;29:102567. http://doi.org/10.1016/j.nicl.2021.102567.

54. Pilato F, Profice P, Ranieri F, Capone F, Di lorio R, Florio L, et al. Synaptic plasticity in neurodegenerative diseases evaluated and modulated by in vivo neurophysiological techniques. Mol Neurobiol. 2012;46(3):563-71. http://doi.org/10.1007/s12035-012-83029.

55. Sabbagh M, Sadowsky C, Tousi B, Agronin ME, Alva G, Armon C, et al. Effects of a combined transcranial magnetic stimulation (TMS) and cognitive training intervention in patients with Alzheimer's disease. Alzheimers Dement. 2020;16(4):641-50. http://doi.org/10.1016/j.jalz.2019.08.197.

56. Lee J, Choi BH, Oh E, Sohn EH, Lee AY. Treatment of Alzheimer's Disease with Repetitive Transcranial Magnetic Stimulation Combined with Cognitive Training: A Prospective, Randomized, Double-Blind, Placebo-Controlled Study. J Clin Neurol. 2016;12(1):57-64. http://doi.org/10.3988/jcn.2016.12.1.57.

57. Scheltens P, De Strooper B, Kivipelto M, Holstege H, Chételat G, Teunissen CE, et al. Alzheimer's disease. The Lancet. 2021. http://doi.org/10.1016/s0140-6736(20)32205-4.

\section{Figures}

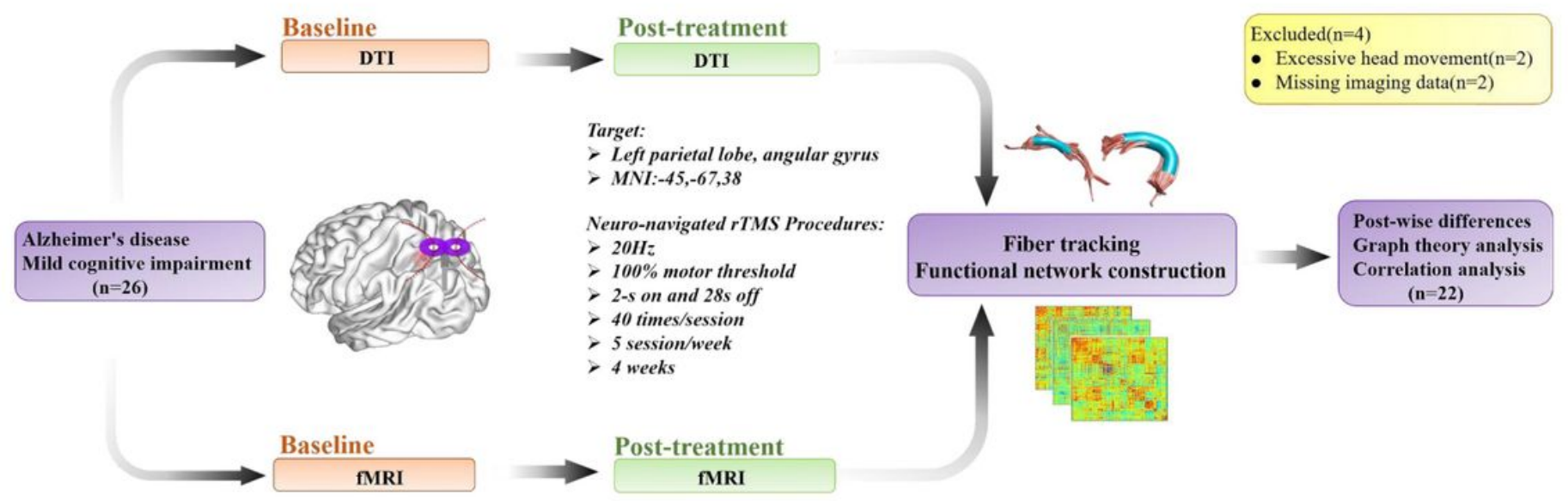

\section{Figure 1}

Study and experiment design All patients $(n=26)$ were stimulated the angular gyrus (MNI: $-45,-67,38)$. by the Neuro-navigated rTMS for four weeks. rTMS was applied at $20 \mathrm{~Hz}$ five times a week, using a neuron avigation system to ensure that the same spot was constantly stimulated across sessions. 


\section{Total}
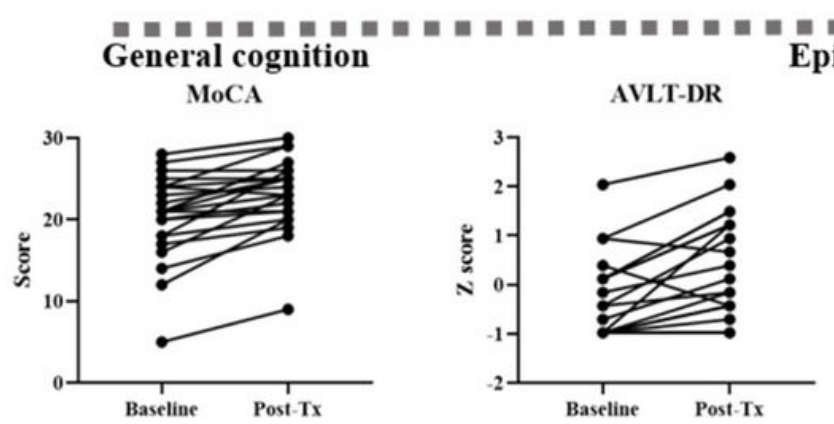

Episodic Memory

VR-DR

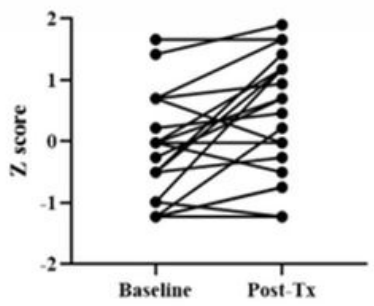

Language

BNT

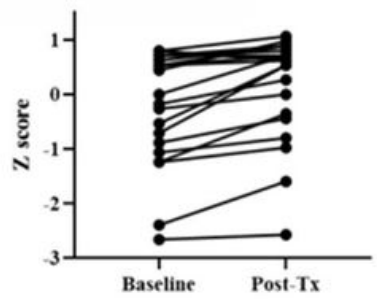

\section{Pre-dementia}

General cognition

MoCA

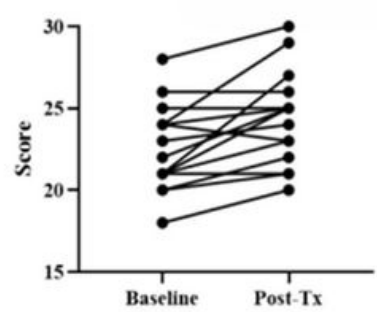

Episodic Memory

AVLT-DR

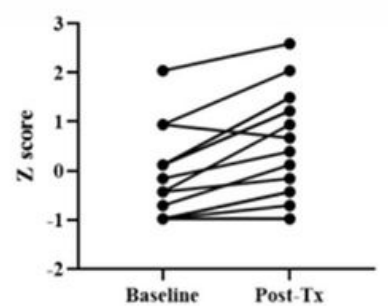

VR-DR

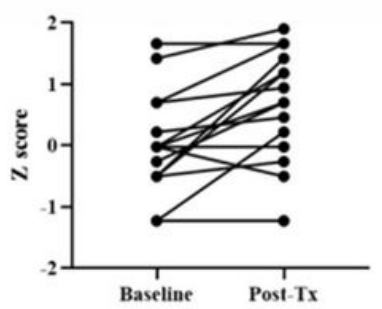

Language

BNT

\section{Dementia}

a

General cognition

MoCA

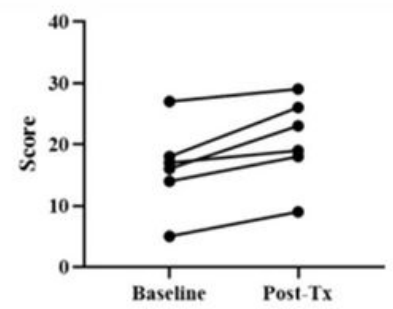

Language

BNT
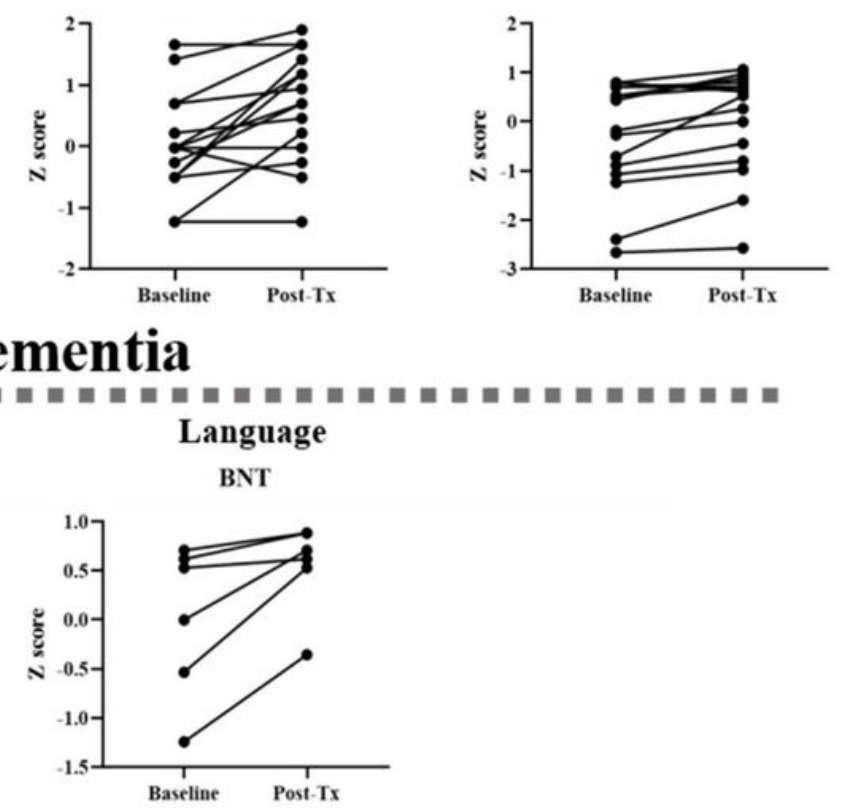

Figure 2

Behavioral result All participants showed improvement in general cognition, episodic memory and language function. MCl's general cognition, episodic memory and language function improved and AD's general cognition, and language function were also improved. (p $<$ 0.05.) Abbreviations: AVLT-DR, Auditory Verbal Learning Test-delayed recall; BNT, Boston Naming Test; CVF, category verbal fluency; MMSE, mini mental state examination; MoCA-BJ, Beijing version of the Montreal Cognitive Assessment; Post-Tx: post-treatment; VR-DR, visual reproduction-delay recall 

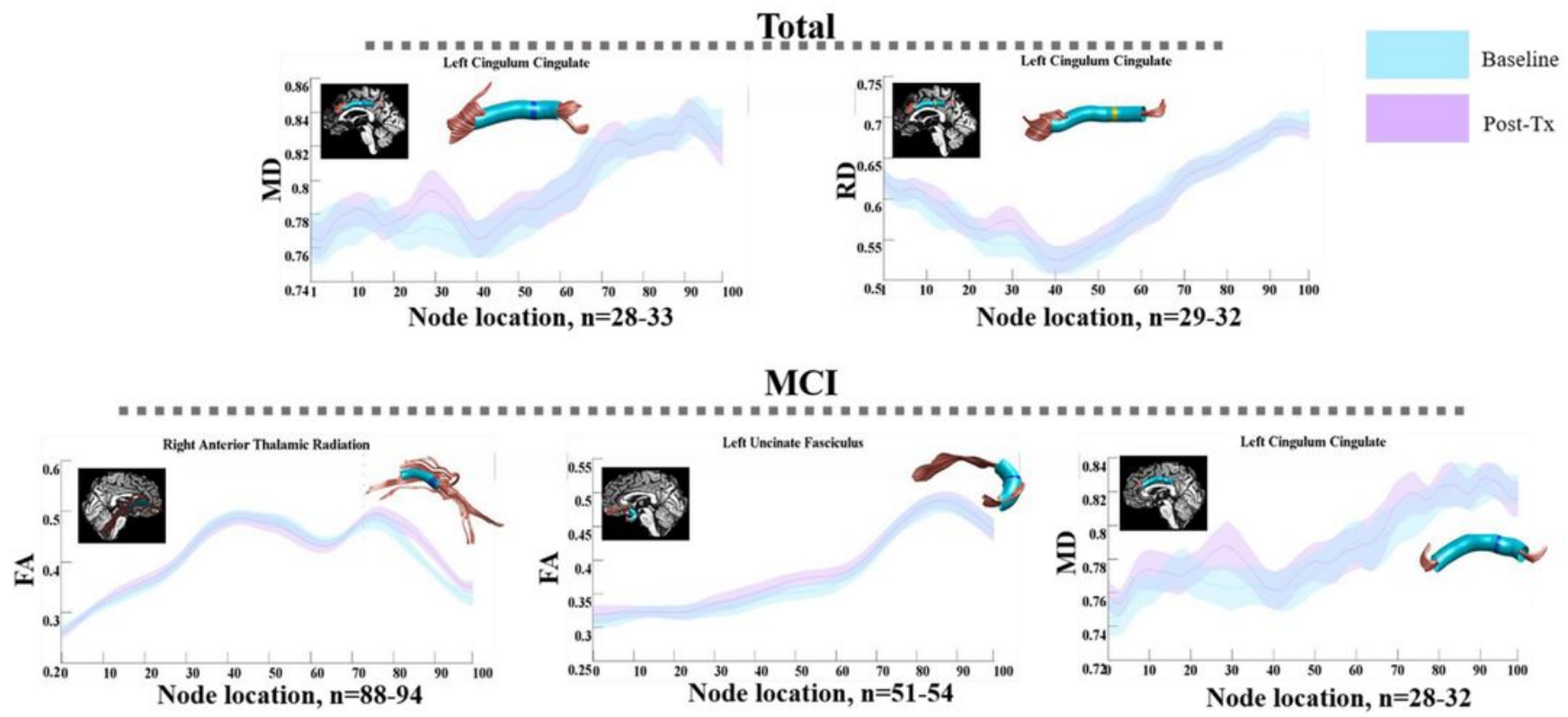

\section{Figure 3}

Post-wise differences in fiber tracts Significantly altered values in point-wise of fiber tracts (FDR correction, $\mathrm{p}<0.05$ ), Dark blue color in the white matter tracts represents significantly altered locations. Light blue color in the white matter tracts represents other locations with no statistical significance. Abbreviations: FA, fractional anisotropy; MD, mean diffusivity; RD, radial diffusivity. 
(A) Nodal Shortest Path Length

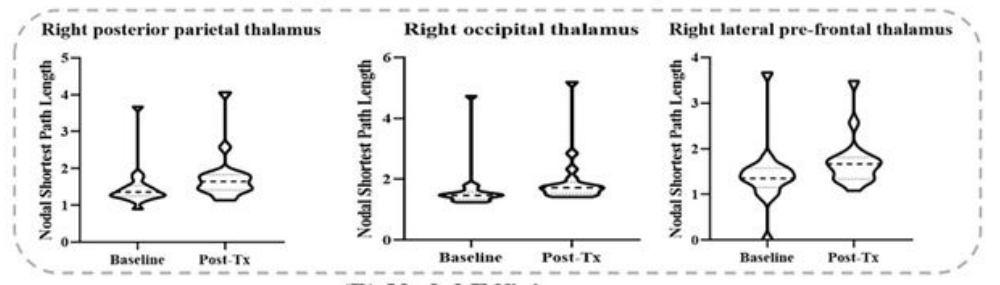

(B) Nodal Efficiency

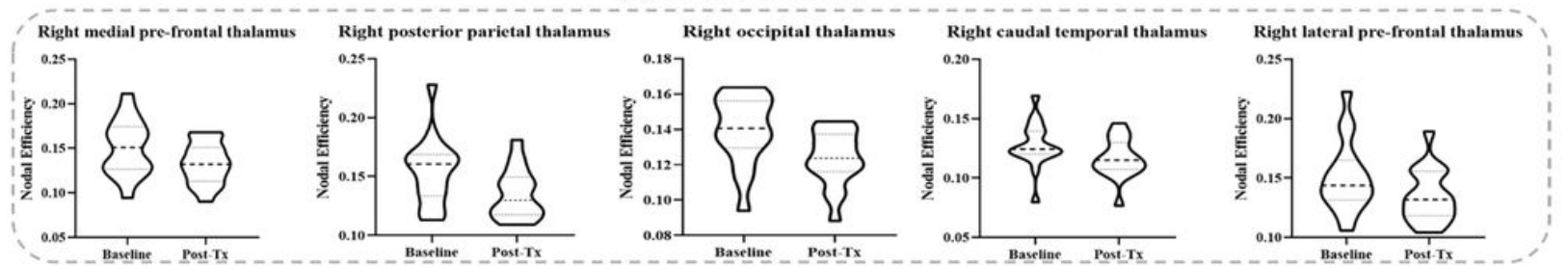

(C) Degree Centrality

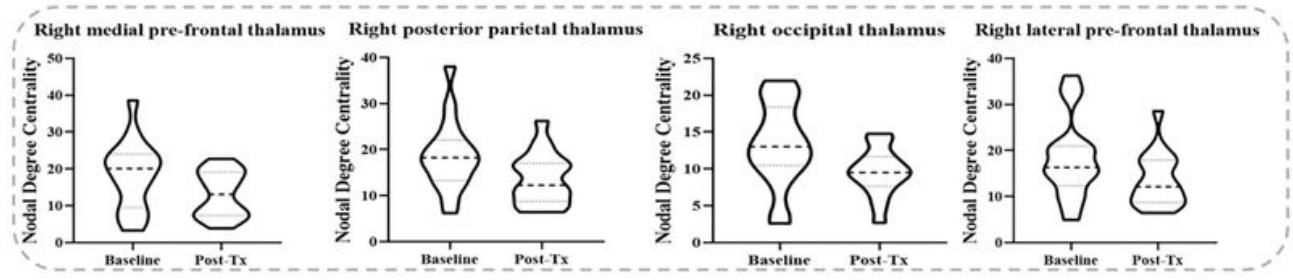

\section{Figure 4}

Alterations in thalamic-related functional network properties In $\mathrm{MCl}$ group, Nodal shortest path length of right posterior parietal thalamus, occipital thalamus and lateral pre-frontal thalamus was improved. (FDR correction, $p<0.05$ ). Nodal efficiency of right medial pre-frontal thalamus, posterior parietal thalamus, occipital thalamus, caudal temporal thalamus, lateral pre-frontal thalamus was decreased. (FDR correction, $\mathrm{p}<0.05$ ). Degree centrality in right medial pre-frontal thalamus, posterior parietal thalamus, occipital thalamus and lateral pre-frontal thalamus was decreased (FDR correction, $p<0.05$ ). 

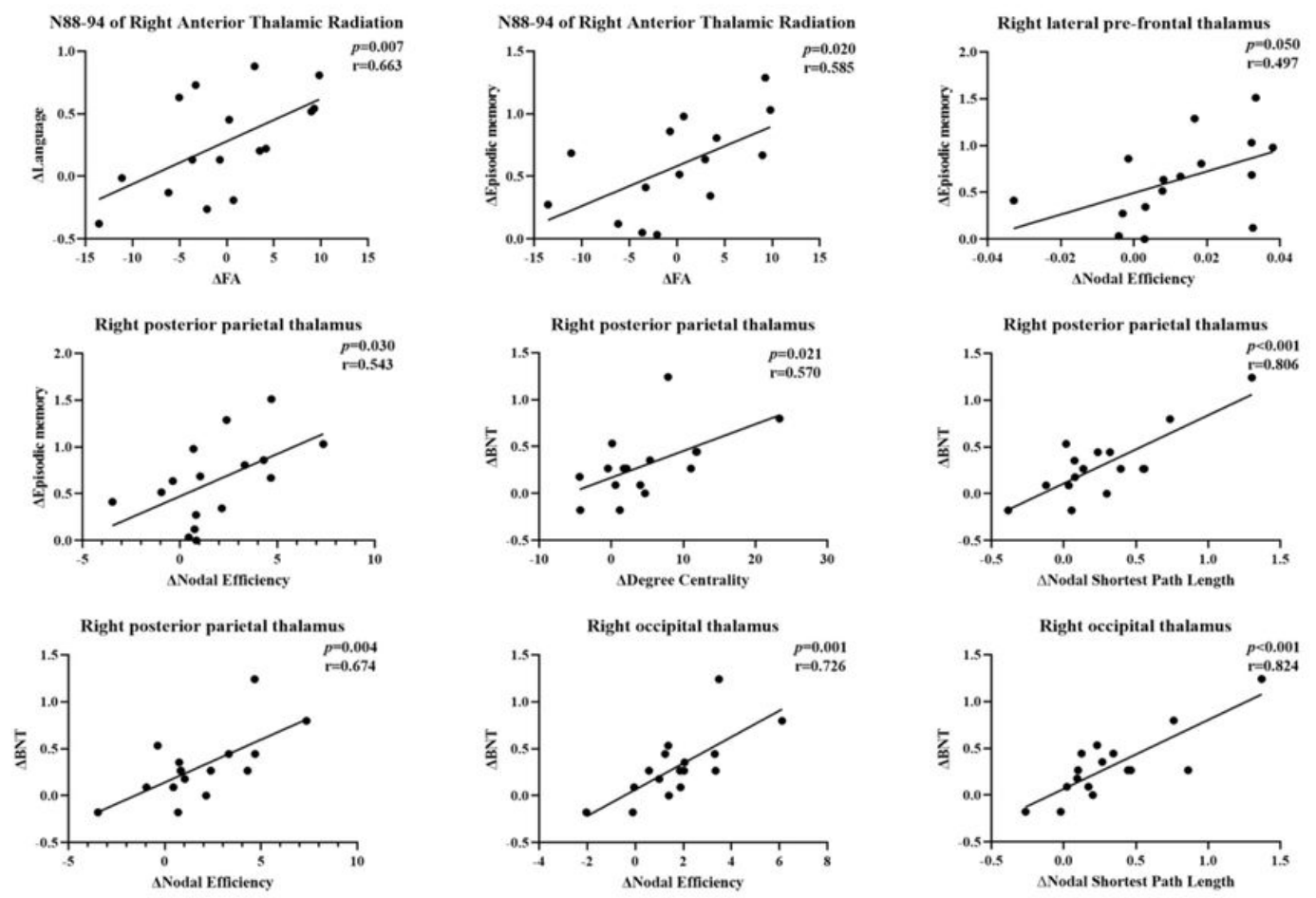

\section{Figure 5}

Correlations between diffusion metrics, network Properties and cognition assessment In MCl group, $\triangle F A$ of N88-94 in right thalamic radiation was positively correlated with improved episodic memory $(r=0.585, p=0.028)$ and language function $(r=0.663, p=0.007)$. Before analysis, we took the negative numbers of these attributes. Nodal efficiency in right posterior parietal thalamus $(r=0.543, p=$ $0.030)$, right lateral pre-frontal thalamus $(r=0.497, p=0.050)$ was positively correlated the improved episodic memory. Degree centrality $(r=0.577, p=0.021)$ and nodal efficiency $(r=0.674, p=0.004)$ of right posterior parietal thalamus, nodal efficiency of right occipital thalamus $(r=0.726, p=0.001)$, nodal shortest path of right posterior parietal thalamus $(r=0.806, p<0.001)$, right occipital thalamus ( $r$ $=-0.824, p<0.001)$ were positively correlated with the score of BNT test

\section{Supplementary Files}

This is a list of supplementary files associated with this preprint. Click to download.

- SupplementaryData.docx 